\title{
BMJ Open Impact of surgical margin status on the survival outcome after surgical resection of gastric cancer: a protocol for systematic review and meta-analysis
}

\author{
Zhiyuan Jiang (D) , ${ }^{1}$ Zhaolun Cai (D) , ${ }^{1}$ Yuan Yin, ${ }^{1}$ Chaoyong Shen, ${ }^{1}$ Jinming Huang, ${ }^{2}$ \\ Yiqiong Yin, ${ }^{1}$ Bo Zhang ${ }^{1}$
}

To cite: Jiang Z, Cai Z, Yin Y, et al. Impact of surgical margin status on the survival outcome after surgical resection of gastric cancer: a protocol for systematic review and meta-analysis. BMJ Open 2020;10:e040282. doi:10.1136/ bmjopen-2020-040282

- Prepublication history and additional material for this paper are available online. To view these files, please visit the journal online (http://dx.doi. org/10.1136/bmjopen-2020040282).

ZJ and ZC contributed equally.

Received 09 May 2020

Revised 05 August 2020

Accepted 17 September 2020

Check for updates

(c) Author(s) (or their employer(s)) 2020. Re-use permitted under CC BY-NC. No commercial re-use. See rights and permissions. Published by BMJ.

${ }^{1}$ Department of Gastrointestinal Surgery, Sichuan University West China Hospital, Chengdu, Sichuan, China

${ }^{2}$ Department of Rehabilitation Medicine, Sichuan University West China Hospital, Chengdu, Sichuan, China

Correspondence to

Professor Bo Zhang;

hxwcwk@126.com and

Yiqiong Yin;

yiqiong489@163.com

\section{ABSTRACT}

Introduction Generally, complete resection with cancer cell negative (R0) margin has been accepted as the most effective treatment of gastric cancer and positive resection (R1/R2) margin has been associated with decreased survival to varied degrees. However, the independent impact of microscopical positive (R1) margin on long-term survival may be confounded. No meta-analysis has worked at the association between R1 margin and outcomes of gastric cancer and the available evidence are scant. Therefore, we plan to conduct a systematic review and meta-analysis to quantitatively explore the role of R1 margin on gastric (including oesophagogastric junction) cancer survival after curative intent resection.

Methods and analysis The protocol was conducted according to Preferred Reporting Items for Systematic Review and Meta-Analysis Protocols guideline. A systematic search of PubMed, Embase and the Cochrane Central Register of Controlled Trials databases will be performed from their inceptions to 30 April 2020 to identify randomised controlled trials (RCTs), cohort studies and case-control studies focusing on the impact of R1 margin on survival of gastric cancer after curative intent resection. The primary outcome will be the overall survival (OS) and diseasefree survival (DFS) and the secondary outcomes will be 5-year OS rate and 5-year DFS rate. The Cochrane tool for bias assessment in randomised trials and Risk Of Bias In Non-randomised Studies-I for the assessment of bias in non-randomised studies (NRS) will be used. Statistical heterogeneity will be assessed by visual inspection of forest plots and measured using the $\mathrm{I}^{2}$ statistics. A fixed-effect model will be used when heterogeneity is low, otherwise, a random-effect model will be chosen. Publication bias will be assessed by funnel plots, subgroup analysis and sensitivity analysis will be performed in the right context. For each outcome, we will perform data synthesis separately for RCTs and NRS using Rev Man V.5.3 software and compile 'summary of findings' tables separately for RCTs and NRS using GRADEpro software. Grading of Recommendations, Assessment, Development and Evaluations considerations will also be used to make an overall assessment of the quality of evidence.

Ethics and dissemination There is no requirement for ethics approval because no patient data will be collected at an individual level in this systematic review and metaanalysis.
Strengths and limitations of this study

- This present protocol will provide transparency to the development process of our systematic review and accountability for the authors such that bias is minimised.

- To the best of our knowledge, this will be the first meta-analysis to explore influence of $\mathrm{R} 1$ margin on gastric cancer survival after curative intent resection.

- Our findings might provide relevant evidence to help surgeons to determine whether their patients with $\mathrm{R} 1$ margins need reresections or other aggressive treatments.

- Risk of bias will be evaluated at both study and outcome levels.

- The quality of evidence and grading of recommendation of our systematic review may be limited by a potential lack of randomised controlled trials that meet the inclusion criteria.

The results of this systematic review will be published in a peer-reviewed journal and presented at relevant conferences, any deviations from the protocol will be clearly documented and explained in its final report. PROSPERO registration number CRD42020165110.

\section{INTRODUCTION}

Gastric cancer is the fifth most frequently diagnosed cancer and the third leading cause of death from cancer in the world. ${ }^{1}$ Although adjuvant therapies can improve outcomes of some patients, surgical resection is considered to be the first line and only possible radical treatment for gastric cancer. $^{2-4}$ Complete resection with cancer cell negative (R0) margin (no cancer cells identified at the resection margin by pathological examination) has been accepted as the most effective treatment based on the surgical philosophy and even minimal remaining cancer cells will develop recurrences. ${ }^{56}$ The recently reported rate of 
positive resection, which included both positive resection (R1) margin (cancer cells presented at the resection margin by pathological examination) and R2 margin (tumour tissue seen at the resection margin on gross examination by the naked eye) was $24.2 \% .^{7}$ Although the influence of R1 margin on gastric cancer survival has been the topics of many studies, inconsistent conclusions have been reached. ${ }^{25-18}$ For example, Kim et al ${ }^{17}$ and Postlewait $e t \mathrm{al}^{14}$ found that R1 margin was not independently associated with survival while Woo $e t a l^{13}$ and Nagata et a $a \tilde{l}$ revealed R1 margin boded ill for survival. Bickenbach et $a l^{11}$ found that R1 margin was an independent predictor of worse survival, but not in patients with $>3$ positive nodes or T3-4 disease. Schoenfeld et $a l^{18}$ found R1 margins were associated with disease-free survival (DFS) but not overall survival (OS).

Raziee et $a l^{19}$ conducted a systematic review in 2012 examining R1 and R2 margins of gastric cancer by exploring their predictive factors, impact on survival and optimal strategies for reresection, but it did not perform meta-analysis. It was still uncertain whether $\mathrm{R} 1$ margin had negative influence on survival and whether it is worth performing reresection to eliminate R1 margin. Therefore, we plan to conduct a systematic review and meta-analysis to quantitatively explore the impact of R1 margin on gastric cancer survival after curative intent resection. Since the preformal study literature search found that original studies on this topic were almost nonrandomised studies (NRS), our systematic review will consider both randomised controlled trials (RCTs) and NRS. The protocol for our research is reported according to the Preferred Reporting Items for Systematic Review and Meta-Analysis Protocols (PRISMA-P), ${ }^{20}$ its PRISMA-P checklist file is attached in online supplemental file 1. The findings of the review will be sought published in a peer-reviewed journal, also in the event of insignificant results or null results, and thereby it will be disseminated to clinicians and public available.

\section{OBJECTIVES}

The aim of this systematic review and meta-analysis is to explore the following:

1. Whether R1 margin negatively influences the survival of gastric cancer after curative intent resection.

2. Which subgroups are most impacted by $\mathrm{R} 1$ margin and which are not?

The study will be conducted based on the following requirements.

\section{Population}

Patients who have undergone curative intent resection for gastric cancer diagnosed by pathological examination will be included.

Gastric cancer patients underwent palliative intent resection will be excluded.

\section{Exposure}

The exposure will be postoperative R1 margin, which means that cancer cells are identified by pathological examination at the linear, circular, proximal or distal resection margin.

Margin status identified as R1 by intraoperative frozen section but R0 by pathological examination and R2 margin will be excluded.

\section{Control}

R0 margin is confirmed by pathological examination.

\section{Outcomes}

Primary outcome: time-to-event OS and time-to-event DFS or relevant data to estimate them.

Secondary outcome: 5-year OS rate and 5-year DFS rate or relevant data to estimate them.

Studies in which relevant data about these outcomes are impossible to extract will be excluded.

\section{METHODS}

This systematic review and meta-analysis will be conducted according to the PRISMA guidelines. ${ }^{22}$

\section{Criteria for considering studies}

\section{Inclusion criteria}

1. RCTs, cohort studies or case-control studies estimating the impact of margin status on gastric cancer survival after curative intent resection.

2. Studies with a minimum of 60 months follow-up reporting time-to-event OS or DFS or reporting 5-year OS rate or 5-year DFS rate.

3. Studies including only human participants.

4. There will be no restrictions on language and publication year.

\section{Exclusion criteria}

1. Studies with overlapping data.

2. Studies researching endoscopic submucosal dissection and endoscopic mucosal resection of gastric cancer.

\section{Search strategy}

The search strategy will be conducted in two stages.

\section{Bibliographic database searches}

A systematic search of PubMed, Embase and the Cochrane Central Register of Controlled Trials databases will be performed from their inceptions to 30 April 2020 to identify all relevant studies. The details of PubMed database search strategy and syntax are shown in table 1.

\section{Searching for other sources}

We will manually search the references of relevant articles to further identify eligible studies, and their full texts will be retrieved.

\section{Study selection and data extraction}

Records identified according to search strategy will be collated and exported to EndNote V.X8 software. Two 


\begin{tabular}{ll}
\hline Table 1 Search strategy for PubMed \\
\hline Search & Search terms \\
\hline \#1 & "Neoplasm, Stomach" or "Stomach Neoplasm" or "Neoplasms, Stomach" or "Gastric Neoplasms" or "Gastric \\
& Neoplasm" or "Neoplasm, Gastric" or "Neoplasms, Gastric" or "Cancer of Stomach" or "Stomach Cancers" \\
& or "Gastric Cancer" or "Cancer, Gastric" or "Cancers, Gastric" or "Gastric Cancers" or "Stomach Cancer" or \\
& "Cancer, Stomach" or "Cancers, Stomach" or "Cancer of the Stomach" or "Gastric Cancer, Familial Diffuse" \\
\#2 & "Excision Margin" or "Excision Margins" or "Resection Margin" or "Margin, Resection" or "Margins, Resection" \\
& or "Resection Margins" or "Surgical Margins" or "Margin, Surgical" or "Margins, Surgical" or "Surgical Margin" \\
& or "Positive Surgical Margins" or "Positive Surgical Margin" or "Surgical Margin, Positive" or "Surgical Margins, \\
& Positive" or "Negative Surgical Margins" or "Negative Surgical Margin" or "Surgical Margin, Negative" or "Surgical \\
& Margins, Negative" or "Tumor-Free Margins" or "Margin, Tumor-Free" or "Margins, Tumor-Free" or "Tumour Free \\
& Margins" or "Tumor-Free Margin" \\
\#1 AND \#2 Limits: Publication date to 2020/4/30, Humans species.
\end{tabular}

reviewers ( $\mathrm{ZJ}$ and $\mathrm{ZC}$ ) will independently screen their titles and abstracts. Subsequently, the two independent reviewers will reassess the full texts of the identified studies, verifying the reasons for inclusion and exclusion. Disagreements will be resolved by team consensus.

Data extraction for included studies will be conducted by two reviewers (ZJ and either ZC or YY) independently using a standardised electronic data extraction form (listed in table 2). This form was piloted by all reviewers. The following data will be extracted from all the included studies: first author, publication year, study design, study period, country(region), male rate, age (mean or median), follow-up (mean or median), sample size, R1 margin rate, R0 margin rate, tumour size, tumour site, tumour stage, histologic grade, type of surgery, lymphadenectomy, neoadjuvant or adjuvant treatments, survival outcomes. If outcomes were reported in multiple data sets, the one with more adjusted confounders will be used. All disputes in the process of data extraction will be resolved through team negotiation.

\section{Dealing with missing data}

To pool HRs of OS or DFS, when an HR and its upper or lower limit of $95 \mathrm{CI}$ are provided by a trial, we can calculate $\operatorname{lnHR}$ (the natural logarithm of HR) and its SE and then merge HRs. When the above data are incomplete, we will attempt to contact the authors to retrieve it. If do not get an effective response in 10 days, we will try to estimate some or all of the lnHR, the logrank observed minus expected events (O-E), the logrank variance and the variance of the $\operatorname{lnHR}$ by indirect methods. ${ }^{23}$ If even these indirect methods cannot be applied, we will consider to generate the necessary statistics from published KaplanMeier curves. ${ }^{23}$ When a trial fails to provide necessary statistics by mentioned methods, it will be omitted from pooling HRs. To pool ORs of 5-year OS rate or DFS rate, we will record data on the total number of participants and the incidence of events in each arm of every trial. When these data in the full text are incomplete, we will contact the authors to extract more, trials that fail to provide these necessary data will be excluded from pooling ORs.

\section{Risk of bias assessment}

Two independent reviewers (ZJ and ZC) will assess the methodological quality/risk of bias of included studies, disagreements will be resolved by discussion, where necessary, in consultation with the third reviewer (YY). For RCTs, the risk of bias will be assessed using the Cochrane Collaboration's risk of bias tool ${ }^{24}$. For NRS, we will use the Risk Of Bias In Non-randomised Studies of Interventions (ROBINS-I) tool ${ }^{25}$. Results of this meta-analyses will be interpreted in light of risk of bias assessment of the included studies.

\section{Measurements}

As for time-to-event outcome, the HR will be used to pool overall effects. Dichotomous outcome will be analysed by calculating the OR. Results will be presented as summary relative effect sizes (HR or OR) with $95 \%$ CIs.

\section{Strategy for data synthesis}

We will import extracted data into Rev Man V.5.3 software (Copenhagen: The Nordic Cochrane Centre, The Cochrane Collaboration, 2014) for data synthesis by the first reviewer and checked by the second one. The overall pooled estimates for the association between resection margin status and survival will be calculated in the metaanalysis. Due to the nature of survival analysis, we will first try to extract HR-related data from each included study and then estimate pooled HRs for OS and DFS with 95\% CI according to methods introduced by Parmar et al and Tierney et al. ${ }^{23}$ If multiple HRs for a same outcome are presented in a paper, we will choose the one adjusted for the greatest number of confounders. ${ }^{27}$ Subsequently, we will try to extract the 5-year OS rate and DFS rate of the two groups from each included study and estimate pooled ORs with $95 \%$ CI. NRS with large sample sizes pooling with small RCTs could dominate the pooled effect estimates, thus we will perform data synthesis separately for RCTs and NRS. Statistical heterogeneity will be assessed by visual inspection of forest plots and measured using the $\mathrm{I}^{2}$ statistics. $\mathrm{I}^{2}<50 \%$ or $\geq 50 \%$ indicates low or high heterogeneity, respectively. A fixed effect model will be used when heterogeneity is low, otherwise, a random 
Table 2 Data extraction form

\section{Study details}

General information

First author

Year of publication

Region

Study period

Study eligibility

Study design

Participants

Exposure

Control

Outcome diagnostic criteria

Confounding variables

Include or exclude Include $\square$ exclude $\square$

Reason(s) for exclusion

Characteristics of included studies

Sample size

R1 margin group

R0 margin group

Data source

Age (mean or median)

Gender (male rate)

Follow-up (mean and range)

(months)

R1 margin rate (\%)

R0 margin rate (\%)

Tumour size $(\mathrm{cm})$

Tumour site

Tumour stage

Histologic grade

Type of surgery

Lymphadenectomy

Neoadjuvant or adjuvant treatment

for R1 margin group

Neoadjuvant or adjuvant treatment

for RO margin group

\section{Subgroups}

Key conclusion(s)

\section{Primary outcomes}

HRs (comparing R1 and R0 group)

with $95 \% \mathrm{Cl}$

\section{OS}

DFS

Relevant data to calculate HRs

OS

DFS

Other data

Continued
Table 2 Continued

\begin{tabular}{ll}
\hline Study details & \\
\hline Second outcomes & R1 group: R0 group: \\
5 year OS rate & R1 group: R0 group: \\
5 year DFS rate & R1 group: R0 group: \\
\hline
\end{tabular}

effect model will be chosen. When substantial heterogeneity is detected, subgroup analysis and sensitivity analysis will be performed to investigate its possible sources. In case of considerable clinical heterogeneity, a narrative review rather meta-analysis will be conducted.

\section{Sensitivity analysis and subgroup analyses}

We will apply the leave-one-out sensitivity analysis to evaluate the robustness of the results.

Subgroup analyses are planned as follows:

1. Study carried out in: Asia and other regions.

2. Tumour stage: early gastric cancer and advanced gastric cancer.

3. Lymphadenectomy: $\leq \mathrm{D} 1$ and $\geq \mathrm{D} 2$.

4. HR (estimating OS or DFS) extracted from: multivariate analysis and univariate analysis.

5. Proximal cancer (tumour in the gastro-oesophageal junction, cardia or fundus).

\section{Publication bias assessment}

Since detecting and overcoming publication bias are problematic and firm guidance is not yet offered, we will use visual inspection of funnel plots to assess publication bias, with results being interpreted cautiously. ${ }^{28} 29$

\section{Quality of the evidence}

Two reviewers (ZC and $\mathrm{YY}$ ) will assess overall quality of evidence for each outcome independently using the five Grading of Recommendations, Assessment, Development and Evaluations (GRADE) considerations. In this approach, direct evidence from RCTs begins at high quality, while observational study begins at low; however, the overall quality will be analysed on five down-grade considerations (study limitations, consistency of effect, imprecision, indirectness and publication bias) and three up-grade considerations (large magnitude of effect, doseresponse relation and plausible confounders or biases), and finally rate it as high, moderate, low or very low. ${ }^{30-37}$ Disagreements will be solved by discussion. Whenever necessary, we will provide all decisions to down grade or up grade the quality of studies with clear arguments in footnotes to aid the reader's understanding of the table and the process.

\section{Presentation and reporting of results}

We will summarise the study selection process by a flow diagram with reasons for exclusions (figure 1). The characteristics of each included study will be tabulated in detail. We will use forest plots to present the pooled 


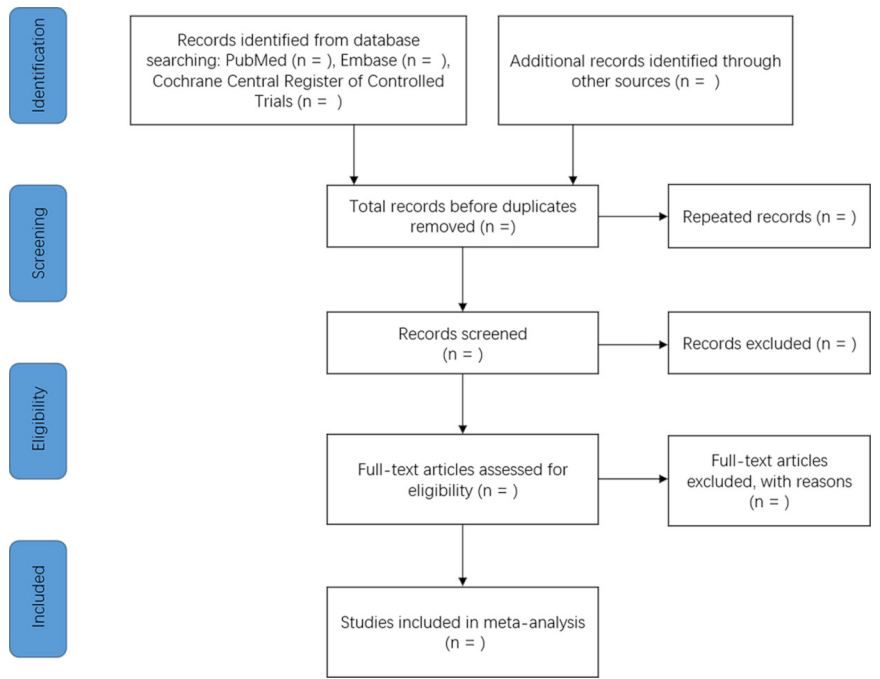

Figure 1 Flow diagram of studies selected for inclusion in this systematic review.

estimates. Additionally, for each outcome, 'summary of findings' tables compiled by GRADEpro software (GRADEpro GDT 2015) will be presented separately for both RCTs and NRS.

\section{Patient and public involvement}

This systematic review and meta-analysis will be based on published studies, raw patient data will not be collected. As a result, patients and the public will not be involved in the design, or conduct, or reporting or dissemination plans of this research.

\section{Ethics and dissemination}

There is no ethics approval required for this systematic review due to no patient data being collected at an individual level. We will seek to present the findings of this systematic review at relevant conferences and publish in an influential open access journal, any deviations from the protocol will be clearly documented and explained in its final report.

\section{SUMMARY}

This is a protocol for a systematic review and meta-analysis reported according to the PRISMA-P guidelines. This systematic review and meta-analysis will explore the influence of R1 margin comparing with $\mathrm{R} 0$ margin on gastric cancer survival by summarising the published studies and reveal which subgroups are most affected by R1 margin and which are not. It will be conducted according to PRISMA guidelines and with robust methodological processes and statistical analyses. Risk of bias will be evaluated at study level using Cochrane Collaboration's risk of bias tool and ROBINS-I tool as well as at outcome level using the GRADE approach. As a result, its findings might provide relevant evidence and help surgeons to determine whether their patients with R1 margins need reresections or other aggressive treatments.
Contributors The original idea of this research was conceived by BZ. JZ and ZC designed the protocol and drafted the manuscript. JZ, ZC, YY and CS participated in developing the eligibility criteria, search strategy, data extraction methods and data summary plan. JZ, ZC and JH will search for studies, extract and analyse the data. BZ and YYQ supervised the work. All authors approved the final version of this manuscript.

Funding This study was funded by the National Natural Science Foundation of China (grant number 81572931) and 1.3.5 project for disciplines of excellence, West China Hospital, Sichuan University (ZYJC18034).

Competing interests None declared.

Patient consent for publication Not required.

Provenance and peer review Not commissioned; externally peer reviewed.

Supplemental material This content has been supplied by the author(s). It has not been vetted by BMJ Publishing Group Limited (BMJ) and may not have been peer-reviewed. Any opinions or recommendations discussed are solely those of the author(s) and are not endorsed by BMJ. BMJ disclaims all liability and responsibility arising from any reliance placed on the content. Where the content includes any translated material, BMJ does not warrant the accuracy and reliability of the translations (including but not limited to local regulations, clinical guidelines, terminology, drug names and drug dosages), and is not responsible for any error and/or omissions arising from translation and adaptation or otherwise.

Open access This is an open access article distributed in accordance with the Creative Commons Attribution Non Commercial (CC BY-NC 4.0) license, which permits others to distribute, remix, adapt, build upon this work non-commercially, and license their derivative works on different terms, provided the original work is properly cited, appropriate credit is given, any changes made indicated, and the use is non-commercial. See: http://creativecommons.org/licenses/by-nc/4.0/.

\section{ORCID iDs}

Zhiyuan Jiang http://orcid.org/0000-0003-2483-3413

Zhaolun Cai http://orcid.org/0000-0002-3706-6703

\section{REFERENCES}

1 Ajani JA, D'Amico TA, Almhanna K, et al. Gastric cancer, version 3.2016, NCCN clinical practice guidelines in oncology. J Natl Compr Canc Netw 2016;14:1286-312.

2 Chen J-D, Yang X-P, Shen J-G, et al. Prognostic improvement of reexcision for positive resection margins in patients with advanced gastric cancer. Eur J Surg Oncol 2013;39:229-34.

3 Liang $Y$, Ding $X$, Wang $X$, et al. Prognostic value of surgical margin status in gastric cancer patients. ANZ J Surg 2015;85:678-84.

4 Cai Z, Yin Y, Yin Y, et al. Comparative effectiveness of adjuvant treatments for resected gastric cancer: a network meta-analysis. Gastric Cancer 2018;21:1031-40.

5 Nagata T, Ichikawa D, Komatsu S, et al. Prognostic impact of microscopic positive margin in gastric cancer patients. J Surg Oncol 2011;104:592-7.

6 Cai Z, Yin Y, Shen C, et al. Comparative effectiveness of preoperative, postoperative and perioperative treatments for resectable gastric cancer: a network meta-analysis of the literature from the past 20 years. Surg Oncol 2018;27:563-74.

7 Koumarianou A, Krivan S, Machairas N, et al. Ten-Year survival outcomes of patients with potentially resectable gastric cancer: impact of clinicopathologic and treatment-related risk factors. Ann Gastroenterol 2019;32:99-106.

8 Cho BC, Jeung $\mathrm{HC}$, Choi HJ, et al. Prognostic impact of resection margin involvement after extended (D2/D3) gastrectomy for advanced gastric cancer: a 15-year experience at a single Institute. J Surg Oncol 2007;95:461-8.

9 Wang S-Y, Yeh C-N, Lee H-L, et al. Clinical impact of positive surgical margin status on gastric cancer patients undergoing gastrectomy. Ann Surg Oncol 2009;16:2738-43.

10 Sun Z, Li D-ming, Wang Z-ning, et al. Prognostic significance of microscopic positive margins for gastric cancer patients with potentially curative resection. Ann Surg Oncol 2009;16:3028-37.

11 Bickenbach KA, Gonen M, Strong V, et al. Association of positive transection margins with gastric cancer survival and local recurrence. Ann Surg Oncol 2013;20:2663-8.

12 Liang $Y$, Ding $X$, Wang $X$, et al. Prognostic value of surgical margin status in gastric cancer patients. ANZ J Surg 2015;85:678-84.

13 Woo J-W, Ryu KW, Park JY, et al. Prognostic impact of microscopic tumor involved resection margin in advanced gastric cancer patients after gastric resection. World J Surg 2014;38:439-46. 
14 Postlewait LM, Squires MH, Kooby DA, et al. The importance of the proximal resection margin distance for proximal gastric adenocarcinoma: a multi-institutional study of the US gastric cancer collaborative. J Surg Oncol 2015;112:203-7.

15 Bissolati M, Desio M, Rosa F, et al. Risk factor analysis for involvement of resection margins in gastric and esophagogastric junction cancer: an Italian multicenter study. Gastric Cancer 2017;20:70-82

$16 \mathrm{Kim}$ BS, Oh ST, Yook JH, et al. Appropriate gastrectomy resection margins for early gastric carcinoma. J Surg Oncol 2014;109:198-201.

17 Kim Y, Squires MH, Poultsides GA, et al. Impact of lymph node ratio in selecting patients with resected gastric cancer for adjuvant therapy. Surgery 2017;162:285-94.

18 Schoenfeld JD, Wo JY, Mamon HJ, et al. The impact of positive margins on outcome among patients with gastric cancer treated with radiation. Am J Clin Oncol 2016;39:243-7.

19 Raziee HR, Cardoso R, Seevaratnam R, et al. Systematic review of the predictors of positive margins in gastric cancer surgery and the effect on survival. Gastric Cancer 2012;15:116-24.

20 Shamseer L, Moher D, Clarke M, et al. Preferred reporting items for systematic review and meta-analysis protocols (PRISMA-P) 2015: elaboration and explanation. BMJ 2015;350:g7647.

21 Moher D, Shamseer L, Clarke M, et al. Preferred reporting items for systematic review and meta-analysis protocols (PRISMA-P) 2015 statement. Syst Rev 2015;4:1.

22 Moher D, Liberati A, Tetzlaff J, et al. Preferred reporting items for systematic reviews and meta-analyses: the PRISMA statement. BMJ 2009;339:b2535.

23 Tierney JF, Stewart LA, Ghersi D, et al. Practical methods for incorporating summary time-to-event data into meta-analysis. Trials 2007;8:16.

24 Higgins JPT, Altman DG, Gøtzsche PC, et al. The Cochrane collaboration's tool for assessing risk of bias in randomised trials. BMJ 2011;343:d5928.
25 Sterne JA, Hernán MA, Reeves BC, et al. ROBINS-I: a tool for assessing risk of bias in non-randomised studies of interventions. BMJ 2016;355:i4919.

26 Parmar MK, Torri V, Stewart L. Extracting summary statistics to perform meta-analyses of the published literature for survival endpoints. Stat Med 1998;17:2815-34.

27 Hunink MG, Wong JB. Meta-Analysis of failure-time data with adjustment for covariates. Med Decis Making 1994;14:59-70.

28 Egger M, Davey Smith G, Schneider M, et al. Bias in meta-analysis detected by a simple, graphical test. BMJ 1997;315:629-34.

29 Li F, Liu G, Roudi R, et al. Do statins improve outcomes for patients with non-small cell lung cancer? A systematic review and metaanalysis protocol. BMJ Open 2018;8:e022161.

30 Balshem H, Helfand M, Schünemann HJ, et al. Grade guidelines: 3. rating the quality of evidence. J Clin Epidemiol 2011;64:401-6.

31 Guyatt GH, Oxman AD, Kunz R, et al. GRADE guidelines 6. Rating the quality of evidence--imprecision. J Clin Epidemiol 2011;64:1283-93.

32 Guyatt GH, Oxman AD, Kunz R, et al. GRADE guidelines: 8. Rating the quality of evidence--indirectness. J Clin Epidemiol 2011;64:1303-10.

33 Guyatt GH, Oxman AD, Kunz R, et al. GRADE guidelines: 7. Rating the quality of evidence--inconsistency. J Clin Epidemiol 2011;64:1294-302.

34 Guyatt GH, Oxman AD, Montori V, et al. GRADE guidelines: 5. Rating the quality of evidence--publication bias. J Clin Epidemiol 2011;64:1277-82.

35 Guyatt GH, Oxman AD, Sultan S, et al. Grade guidelines: 9. rating up the quality of evidence. J Clin Epidemiol 2011;64:1311-6.

36 Guyatt GH, Oxman AD, Vist G, et al. GRADE guidelines: 4. Rating the quality of evidence--study limitations (risk of bias). J Clin Epidemiol 2011;64:407-15.

37 Kien C, Gartlehner G, Kaminski-Hartenthaler A, et al. [GRADE guidelines: 9. Rating up the quality of evidence]. Z Evid Fortbild Qual Gesundhwes 2013;107:249-55. 